\title{
ANÁLISIS DE LAS NECESIDADES SOCIALES, EDUCATIVAS Y DE ORIENTACIÓN DE LAS CUENCAS MINERAS DE ASTURIAS
}

\author{
ANALYSIS OF THE SOCIAL, EDUCATIONAL AND GUIDANCE NEEDS \\ OF THE MINING ZONES FROM ASTURIAS
}

\author{
Marisa Pereira González* y Julián Pascual Díez**
}

\begin{abstract}
RESUMEN
En este artículo se presentan los principales resultados de una investigación que tiene por objeto analizar las necesidades sociales, educativas y de orientación de las cuencas mineras del Principado de Asturias. En primer lugar, se incluye una breve referencia a la metodología del estudio, en el que se elaboró un dossier de prensa, se efectuaron entrevistas a expertos analizadores sociales y se realizaron grupos de discusión con jóvenes y familias de las cuencas mineras. Posteriormente, se exponen las conclusiones sobre los principales problemas y propuestas formuladas en cada área: educación, demografía y empleo; jóvenes; mujeres; personas mayores y personas con discapacidad. Por último, se plantean las propuestas socioeducativas que se sugieren para tratar de responder a las necesidades de estas zonas en crisis.
\end{abstract}

Palabras clave: Evaluación de necesidades sociales, educativas y de orientación, cuencas mineras asturianas.

\begin{abstract}
In this article we present the main results of the research that we carry out in order to analyze the social, educational and guidance needs of the mining zones from Asturias. First we refer shortly to the methodology of the study in which we elaborated a newspapers dossier, we interviewed many social experts and we organized discussion groups with youth and families of the mining zones. Then we expose the conclusions about the main problems and proposals to resolve them in each area: education, demography and employment, youth, women, aged people and people with disabilities. Finally, we include the social and educational proposals that are suggested in order to respond to the needs of these areas in crisis.
\end{abstract}

Key words: Evaluation of social, educational and guidance needs, mining zones from Asturias.

* Marisa Pereira González es Profesora Titular del Departamento de Ciencias de la Educación (MIDE).

** Julián Pascual Diez es Profesor Titular del Departamento de Ciencias de la Educación (Didáctica de la Lengua). Universidad de Oviedo, Facultad de Ciencias de la Educación, C/ Aniceto Sela s/n, 33005, Oviedo. Tel. 985.103242, E-mail: pereira@uniovi.es; jpascual@uniovi.es. 


\section{Introducción}

Este trabajo forma parte de un proyecto más amplio, compuesto por tres estudios realizados con objeto de:

1) describir las características socioeducativas de las cuencas mineras de Asturias;

2) analizar las demandas y necesidades socioeducativas en estas comarcas y

3) analizar las condiciones y recursos de FUNDOMA (Fundación Docente de Mineros Asturianos) para identificar posibles alternativas viables para dicha institución educativa.

Nuestro equipo de trabajo se ha encargado del segundo estudio, cuyos resultados presentamos en este artículo ${ }^{2}$. Debe tenerse en cuenta que, debido a las limitaciones de espacio, es imposible pormenorizar los resultados de todas las fases de la evaluación que realizamos, las cuales están contenidas en amplios Informes. En este caso hemos preferido centrarnos en las necesidades más significativas vinculadas a las distintas áreas sociales analizadas - porque reflejan claramente la realidad de las cuencas mineras asturianas-, en las propuestas de mejora vinculadas a cada área, para concluir con las principales implicaciones prácticas derivadas del estudio. Buena parte de la solución de los problemas evidenciados, como veremos, requiere considerables esfuerzos de formación y orientación.

\section{Método}

Esta investigación ha sido realizada por un equipo de profesores del Departamento de Ciencias de la Educación de la Universidad de Oviedo, motivada por la importancia de la situación de crisis económica y social que se vive en las cuencas mineras asturianas. El objetivo del trabajo es conocer, analizar y priorizar las principales necesidades socioeducativas en las cuencas mineras y establecer diversas propuestas para afrontar los problemas y carencias detectados.

Este estudio se tipifica como una evaluación de las necesidades socio-educativas de las cuencas mineras (véanse Mc Killip 1987, Porteus, 1996, Wiener y cols, 1994, Witking, 1994, Witking y Altschuld, 1995). En él se combinan metodologías de trabajo cuantitativas y cualitativas que implican la utilización de diversas fuentes de información y de procedimientos complementarios de recogida y análisis de datos. Las fuentes de información empleadas son:

1) recursos bibliográficos, libros, artículos, informes, documentos, etc.;

2) prensa regional asturiana (noticias que comprenden un período de dos años y medio sobre las cuencas mineras);

3) analizadores sociales: informantes clave que viven o trabajan en las cuencas mineras, o que por su responsabilidad política o institucional conocen muy bien su problemática;

\footnotetext{
1 El proyecto global ha sido dirigido por Mario de Miguel. En el trabajo de campo del segundo estudio han participado Ana Belén Rodríguez, Liliana Ceballos, Ana María Fernández, Andrea Patallo, Laura Sal, Silvia Suárez y Silvia Viso. Destacamos especialmente la colaboración de Ana Belén Rodríguez Suárez en la elaboración del Dossier de Prensa.
} 
4) jóvenes de las tres cuencas mineras; y

5) familias de estas zonas (padres y madres).

Los procedimientos de recogida de información empleados son:

1) identificación, localización y análisis de información obtenida a partir de diversas fuentes documentales (elaboración de un dossier de prensa a partir de noticias sobre los problemas socio-educativos de las cuencas mineras, correspondientes a un período de dos años y medio y publicaciones de libros, revistas y documentos periódicos, informes, folletos divulgativos e información localizada a través de Internet.);

2) cuestionario semi-estructurado elaborado específicamente para realizar las entrevistas en profundidad a los diversos analizadores sociales (profesorado, personal sanitario, políticos, sindicalistas, asistentes sociales, miembros de asociaciones, etc.);

3) grupos de discusión realizados con jóvenes y con familias de las tres cuencas mineras (Caudal, Nalón, Narcea).

El análisis documental y del dossier de prensa, recogido en un informe previo, permitió identificar las dimensiones que conformaron el cuestionario semi-estructurado que elaboramos para realizar las entrevistas en profundidad en una segunda fase de la investigación. También posibilitó la delimitación de las variables que componen cada una de estas dimensiones y facilitó la concreción de los analizadores sociales pertinentes para cada uno de los ámbitos del estudio. Partiendo de un procedimiento de muestreo propositivo, realizamos un total de $\mathbf{3 6 7}$ entrevistas en profundidad a expertos analizadores sociales. La distribución de la muestra generadora de datos es equilibrada en función de las variables de clasificación consideradas (número de habitantes de cada una de las cuencas y sexo y edad de los entrevistados). También se han llevado a cabo 6 sesiones de trabajo mediante grupos de discusión con jóvenes y con familias de las tres cuencas mineras de Asturias.

Los análisis efectuados con la información procedente de las diversas fuentes pueden clasificarse en cuatro categorías:

1) análisis documental del dossier de prensa elaborado y del resto de las publicaciones e información localizada sobre las cuencas mineras;

2) análisis estadísticos descriptivos de los datos obtenidos sobre las variables de información y de clasificación incluidas en el cuestionario semi-estructurado que se aplicó en la realización de las entrevistas (frecuencias, porcentajes, medias);

3) análisis relacionales para comprobar el grado de asociación entre las variables de estudio y las variables de clasificación (análisis de contingencias: ji-cuadrado y razón de verosimilitud);

4) análisis de contenido de los datos cualitativos obtenidos mediante las preguntas abiertas de las entrevistas y en las diferentes sesiones de los grupos de discusión.

Los procedimientos para contrastar la información recabada implican la utilización de sistemas de control basados en análisis estadísticos, en el análisis y categorización de información cualitativa y en la triangulación de la información procedente de las distintas fuentes enunciadas.

En conjunto, se han elaborado dos Informes que recogen: 
1) el análisis documental y del dossier de prensa y

2) el trabajo de campo efectuado con los analizadores sociales, los jóvenes y las familias de las cuencas mineras.

La presentación del complejo proceso metodológico llevado a cabo para efectuar este estudio la detallamos en otro artículo (Pereira y Pascual, 2004).

A continuación, sintetizamos las principales conclusiones del trabajo, integrando la información cuantitativa y cualitativa recabada mediante las entrevistas y los grupos de discusión en relación a cada uno de los ámbitos estudiados. Presentamos en cada apartado tanto los principales problemas, como las propuestas que se formulan para tratar de resolverlos.

\section{Resultados}

A todos los analizadores sociales se les pregunta por la relevancia que en sus zonas atribuyen a los distintos grupos de problemas que se plantean en el cuestionario, elaborado a partir del análisis del dossier de prensa. Los problemas que consideran más preocupantes, con una clara diferencia respecto a los demás, son los relacionados con la demografía y el empleo. En segundo lugar, señalan los problemas de los jóvenes de las cuencas. El tercer rango, con el mismo nivel de importancia, lo ocupan los problemas educativos y los de las mujeres. El cuarto lugar corresponde a los problemas de las personas mayores y el quinto, a los de las personas con discapacidad. En el otro extremo, se sitúan los problemas que menos parecen preocupar en relación con el conjunto: los relacionados con medio ambiente, sanidad y seguridad y protección pública. A continuación, analizamos con detalle los seis ámbitos que han sido considerados más preocupantes.

\section{Principales problemas educativos}

En las comarcas mineras, el nivel educativo que presenta un mayor número de problemas considerados "muy importantes" es el de la Educación Secundaria Obligatoria. Como aspectos más significativos, se destaca, por parte de un $76.3 \%$ de los analizadores sociales, el escaso rendimiento y fracaso escolar de los estudiantes, así como su falta de motivación (71.2\%). También se señala la frecuencia con la que aparecen dificultades de aprendizaje (65.3\%) y el limitado dominio de habilidades comunicativas básicas (lectura, expresión oral y escrita...) por parte del alumnado (64.4\%). Además, se considera insuficiente la oferta de Ciclos Formativos de Grado Medio y Superior de Formación Profesional en las Cuencas (58.5\%) (Fig. 1).

Las respuestas que los analizadores sociales dan a las preguntas abiertas relacionadas con la educación se refieren a problemas generales de cuatro tipos:

- En primer lugar, se recogen problemas y necesidades relacionados directamente con el profesorado: inestabilidad laboral, incremento de bajas por depresión, escasez de motivación e incentivos a su labor, enfrentamiento entre escuela pública y concertada.

- En segundo lugar, se plantean problemas relacionados con el alumnado: absentismo y abandono escolar, disminución en el número de alumnos, falta de motivación y de 


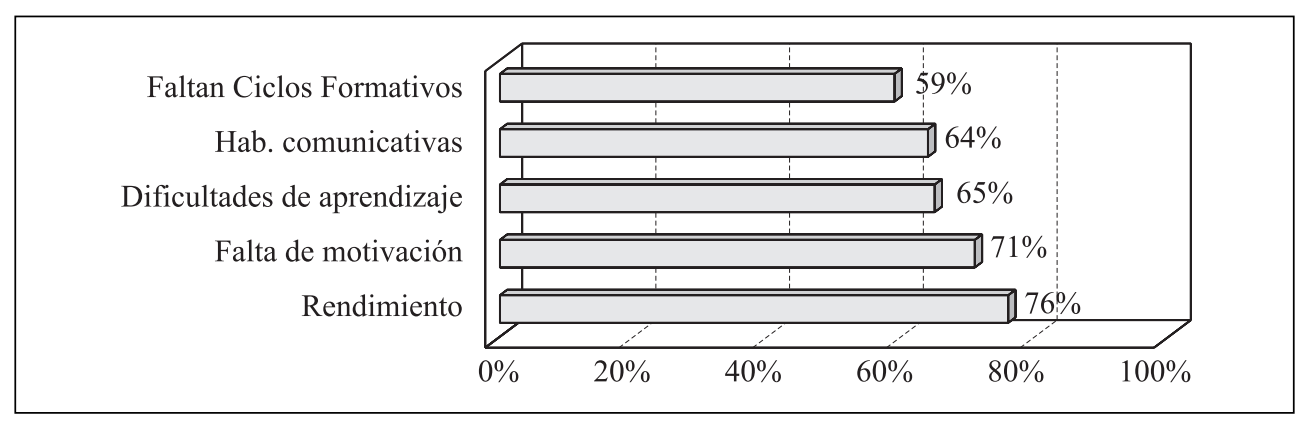

FIGURA 1.

Principales problemas educativos en las cuencas mineras.

interés del alumnado, desarrollo de conductas violentas y machistas, excesivo consumo de televisión, escasas habilidades sociales y de comunicación, escasa variedad de ciclos formativos y falta de adecuación de los mismos.

- En tercer lugar, se alude a problemas relacionados con los padres; sobre todo, se insiste en la falta de implicación de éstos en la educación de sus hijos y en la escasa colaboración que muchos muestran con las actividades propuestas desde los centros escolares.

- Finalmente, se formulan demandas relacionadas con una mayor dotación de infraestructuras, recursos y programas educativos, que resultan plenamente coincidentes con las respuestas de carácter cuantitativo y de las que frecuentemente se hace eco la prensa.

Las propuestas de mejora formuladas en este apartado son muy variadas; las más representativas son:

- Propuestas relacionadas con una potenciación o cambio en la estructura educativa: impulsar la educación en la etapa de 0-3 años; implantar una nueva reforma educativa que supere la ineficacia derivada de la LOGSE, sobre todo, las deficiencias detectadas en la etapa de la ESO; adaptar las especialidades de los ciclos formativos de grado medio a la evolución del mercado laboral y realizar una mayor oferta de ciclos formativos adaptados a las necesidades de la zona; conceder una mayor libertad a los centros educativos para que elaboren su Proyecto Educativo de Centro; analizar y atajar las razones del éxodo de alumnado hacia la escuela privada.

- Propuestas relacionadas con los contenidos educativos: se plantea con carácter general la necesidad de introducir medidas que contribuyan a mejorar el nivel formativo (en Educación Infantil, Primaria y ESO), ya que un grupo significativo de docentes considera que los alumnos finalizan hoy la educación obligatoria con graves carencias; inculcar a los alumnos hábitos de esfuerzo y de trabajo y concienciarles sobre la trascendencia de su formación; trabajar más en los centros la educación en valores.

- Propuestas que se refieren a la necesidad de contar con más medios y recursos: incrementar el número de profesores especialistas en las diferentes áreas; introducir medidas adecuadas para afrontar la atención a la diversidad; aumentar igualmente, el número de orientadores ante la necesidad de abordar de forma más efectiva los pro- 
blemas derivados de las dificultades de aprendizaje, indisciplina, absentismo y abandono y, también, con el fin de asesorar a las familias; evitar la tendencia al agrupamiento comarcal de alumnos y apoyar más a la escuela rural; mejorar notablemente el servicio de transporte escolar, especialmente, en las zonas más alejadas de los núcleos escolares.

- Propuestas que aluden a la formación y a la implicación del profesorado: potenciar la formación permanente del profesorado; introducir medidas que favorezcan una mayor motivación e implicación de los profesores tutores en la labor educativa, sobre todo, en la Educación Secundaria.

- Propuestas que hacen hincapié en una mayor interrelación y comunicación entre los miembros de la comunidad educativa y entre éstos y la sociedad (apertura de los centros a la comunidad): potenciar las relaciones entre padres y profesores; conseguir una mayor implicación de la familia en la vida escolar; impulsar la comunicación entre los centros de enseñanza y las empresas; crear escuelas de padres; abrir los centros educativos a la comunidad; mejorar la coordinación entre servicios sociales y centros educativos; impulsar programas de prevención de alcohol y drogas en jóvenes.

\section{Problemas demográficos y de empleo}

Estos problemas son, por su trascendencia y gravedad, los que más preocupan actualmente en las cuencas mineras.

1. Todos los problemas demográficos, ya identificados en la revisión documental y del dossier de prensa, son muy importantes para la mayoría de los entrevistados: el envejecimiento de la población $(90 \%)$, el éxodo de las cuencas a las principales ciudades del Principado (86.6\%) y la escasa natalidad en las comarcas mineras (75\%).

2. Los problemas relacionados con el empleo que se están produciendo en las comarcas mineras son considerados muy preocupantes por sí mismos y porque, además, generan otros, de carácter social. En primer lugar, se destaca enormemente (ya que es subrayado por un $96.7 \%$ ), la situación generada por la crisis de la minería, lo que ha supuesto la desaparición de muchos puestos de trabajo en estas zonas. Este hecho actúa como un factor desencadenante de un proceso que extiende la crisis a todos los ámbitos sociales (descenso de la población activa, éxodo de la población a otras zonas, elevado número de prejubilaciones, pérdida de mano de obra cualificada, etc.) y se ha visto agravado, aún más, como consecuencia del descenso de la natalidad y el progresivo envejecimiento de la población. A ello se unen los elevados niveles de inactividad en la zona, puesto que la población inactiva no sólo no tiene un empleo, sino que además, ni lo busca activamente, ni hace gestiones para generar su propio empleo, ni está dispuesta a incorporarse al mercado laboral (86.7\%). Además, la crisis de las cuencas se ve agravada por las escasas ofertas de empleo en la zona $(85 \%)$ y por las pocas iniciativas de creación de nuevas empresas $(85 \%)$.

Otros problemas de gran relevancia en las zonas mineras, contribuyen a dibujar — según los especialistas en empleo - un panorama del mercado laboral poco prometedor: al elevado desempleo (destacado por el 75\%), se une el reducido número de iniciativas de autoempleo $(78.3 \%)$ y la magnitud de los problemas de formación existentes. 
Estos problemas de formación vinculados al mundo laboral afectan tanto a los desempleados, como a las personas que ya tienen un empleo. Son problemas de formación: el desajuste entre el perfil formativo de los desempleados y las demandas del mercado laboral (78.4\%); la falta de preparación para incorporarse al mercado laboral (70\%); el desajuste entre la formación de los trabajadores en activo y las nuevas necesidades del mundo laboral (73.4\%); el que los trabajadores no cuenten con las habilidades que se buscan en el mercado laboral - trabajo en equipo, iniciativa emprendedora, creatividad, habilidades sociales, habilidades de negociación...- $(66.7 \%)$ y su falta de conocimiento para manejar las nuevas tecnologías (70\%), aspecto que se une a las deficiencias en la incorporación de las nuevas tecnologías en el empleo (71.7\%). También es importante el desconocimiento de los nuevos yacimientos de empleo (60\%) (Fig. 2).

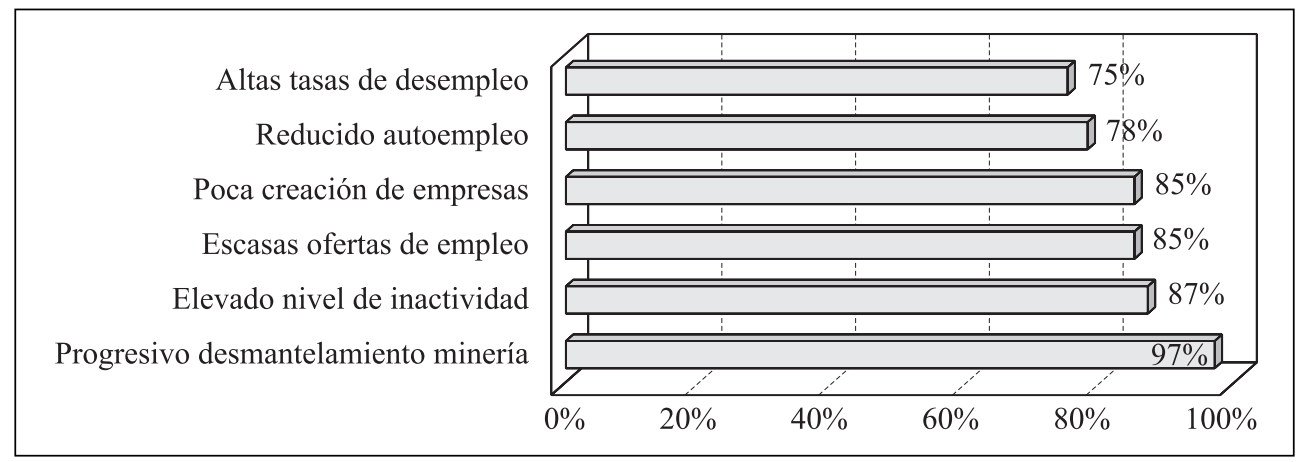

FIGURA 2.

Problemas relacionados con el empleo.

Entre las propuestas que los analizadores sociales formulan para abordar algunos de los problemas relacionados con el empleo destacan las siguientes:

- Llevar a cabo una mayor inversión pública para realizar una auténtica reconversión industrial que favorezca el desarrollo de los nuevos yacimientos de empleo, a través del fomento de diversas alternativas (como puede ser el autoempleo, el empleo asociativo) y la oferta de una serie de servicios y beneficios a las empresas para que se instalen en los polígonos industriales de la comarca: mejorar las infraestructuras, abaratar el suelo industrial, incentivar fiscalmente a los empresarios que quieran invertir, aprovechar los activos ociosos, etc.

- Impulsar la industria turística, hostelería, naturaleza (sectores que puedan tener mejores expectativas).

- Adecuar la formación a la oferta actual del mercado laboral. En este sentido, se formula la propuesta de impulsar una estrecha colaboración entre la Formación Profesional y la Formación Ocupacional que dé respuesta a las posibilidades que se derivan de los nuevos yacimientos de empleo.

- Fomentar las iniciativas emprendedoras y el autoempleo, así como la orientación para el trabajo desde la escuela. Se considera que es preciso modificar la mentalidad labo- 
ral de dependencia de la empresa pública y asumir la necesidad de un cambio socioeconómico y cultural.

- Luchar para asegurar la continuidad de las ayudas procedentes de los Fondos Europeos durante los próximos años.

- Adoptar medidas que favorezcan especialmente la formación de aquellos colectivos en riesgo de exclusión social.

- Agilizar los trámites para hacer funcionar una empresa de nueva creación.

\section{Problemas de los jóvenes}

Los problemas relacionados con los jóvenes son, después de los relacionados con la demografía y el empleo, los considerados más relevantes (Fig. 3).

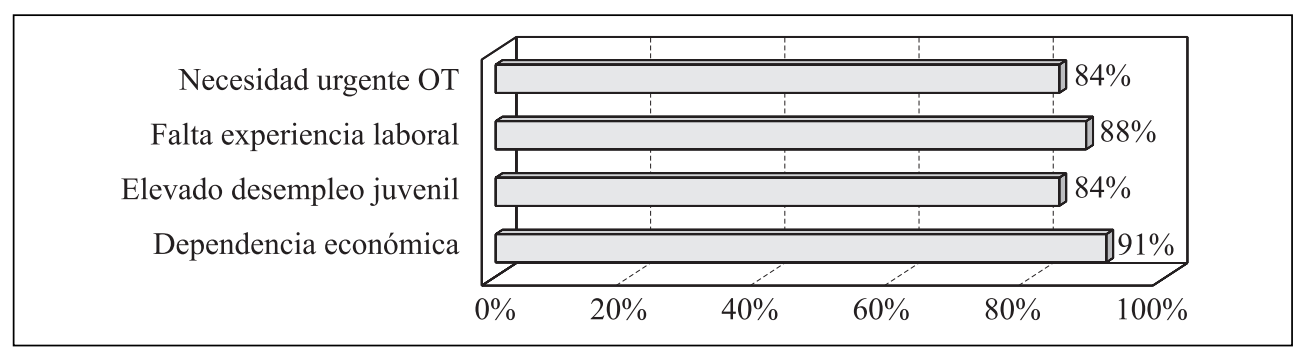

FIGURA 3.

Problemas de los jóvenes.

Los problemas que más preocupan se vinculan a las limitadas posibilidades de acceso al mercado laboral por parte de los jóvenes, condicionadas por la importante crisis económica de las zonas mineras. La principal necesidad es su dependencia económica de la familia y la tardanza en poder independizarse $(90.6 \%)$. Este problema se relaciona con los elevados niveles de desempleo juvenil (84.4\%), con la falta de experiencia laboral que les dificulta el acceso al empleo (87.6\%) y con la urgente necesidad de orientación para el trabajo; necesidad insatisfecha en un altísimo grado (84.4\%). Este conjunto de problemas - tal y como se señala en las entrevistas - contribuye a crear en muchos jóvenes unas expectativas de futuro escasamente halagüeñas, lo que frecuentemente deriva en actitudes caracterizadas por la apatía, la comodidad y la desmotivación y produce el éxodo, en busca de empleo, a otras zonas de la región o de fuera de Asturias. En algunos casos, también es la causa directa del fracaso escolar y de la falta de interés por participar en actividades socioculturales.

También se insiste, aunque en menor medida, en la escasa inversión en políticas de juventud en la zona (75.1\%) y en la falta de espacios de encuentro, reflexión y debate sobre los problemas de los jóvenes (71.9\%). Asimismo, los analizadores sociales entrevistados ponen de manifiesto los problemas relacionados con los hábitos de tiempo libre, destacando la dependencia del tabaco y del alcohol para la diversión con los amigos (71.9\%), el excesivo consumo televisivo $(68.8 \%) \mathrm{y}$, también, un consumo considerable de drogas ilegales 
("porros", drogas de síntesis, cocaína...) (59.4\%). En relación con estos aspectos, se insiste en la insuficiente oferta de transportes públicos nocturnos que se ofrecen para evitar desplazamientos peligrosos, fruto de la combinación de "coche + alcohol".

Las propuestas de los analizadores sociales ante los problemas de los jóvenes son:

- Estimular la creación de puestos de trabajo y la búsqueda de nuevos yacimientos de empleo. En esta línea, se propone la conveniencia de potenciar el autoempleo y el cooperativismo y la orientación para el trabajo.

- Adaptar la formación de los demandantes de empleo a la oferta del mercado y a las necesidades de la zona y ofertar nuevas ramas de especialización.

- Mejorar o crear nuevas infraestructuras para desarrollar programas específicos de ocio alternativo, así como incrementar las subvenciones y los mecanismos de información en este ámbito.

- Crear una política integral de juventud que implique a todos los agentes sociales, con suficientes recursos y apoyos institucionales, que contribuya a impulsar programas de dinamización de las zonas mineras.

- Conceder facilidades para la adquisición y alquiler de vivienda en el colectivo de jóvenes.

- Realizar una mayor inversión en la educación básica para que en esta etapa educativa se comiencen a abordar muchos de los problemas educativos y sociales que posteriormente tendrían una solución más difícil y costosa. Se destaca la necesidad de un mejor trabajo con las familias de los niños y jóvenes y la utilidad de diseñar programas de apoyo y potenciación de la orientación educativa.

\section{Problemas de las mujeres}

Los problemas de las mujeres también ocupan un lugar destacado: las personas que participaron en este estudio los sitúan en el tercer puesto en su orden de prioridades, junto con los de tipo educativo. Los problemas fundamentales tienen que ver con la participación de las mujeres en el mercado laboral, enormemente complicada debido a la grave crisis existente en las zonas mineras. Todos los entrevistados (el 100\%) destacan lo difícil que les resulta tratar de reincorporarse al mercado laboral, una vez que sus hijos son mayores, a causa de una formación que, en muchos casos, resulta inadecuada. Los elevados índices de desempleo femenino (94.2\%) se ven agravados por la falta de experiencia laboral, que les dificulta la consecución de un empleo (94.1\%) y por los problemas que tienen para dejar a sus hijos bien atendidos, mientras trabajan, por falta de guarderías y centros de educación infantil (94.2\%). Preocupan también enormemente, los elevados índices de inactividad femenina en las cuencas, de modo que muchas mujeres ni siquiera se plantean la posibilidad de conseguir un empleo y se dedican principalmente a las labores del hogar (94.1\%). Todo ello pone de relieve la urgencia de una orientación para el trabajo específicamente dirigida a este colectivo (94.1\%).

Las dificultades en la incorporación de la mujer al mercado laboral en las cuencas son muy grandes y se ven agravadas por un bajo nivel formativo, que limita aún más, sus posibilidades de acceso al mundo laboral (88.2\%). Además, también se ve condicionado 
porque existen muchos estereotipos sexistas sobre la implicación de la mujer en el mundo del trabajo (88.2\%). Todo esto lleva a que muchas, que consiguen acceder a un empleo, ocupen puestos de bajo nivel de cualificación (88.3\%). Otro problema, muy relacionado con los anteriores, es que están infra-representadas en la mayoría de los empleos de la zona (82.4\%) y que, igualmente, su representación social y política en las cuencas es muy escasa $(82.4 \%)$.

Por último, es necesario subrayar la preocupación por la violencia doméstica $(82.4 \%)$, que contrasta con la menor relevancia que concede al problema el personal de las fuerzas de seguridad y protección pública. Las personas que específicamente trabajan con el colectivo de mujeres plantean la importancia de la violencia doméstica (física y psicológica) en las cuencas, argumentando que se produce en muchas más ocasiones de las que se denuncia, lo que a veces no se hace por razones de miedo, sentimiento de vergüenza, falta de apoyo de la propia familia, etc. Este problema se relaciona también con la dependencia económica del marido o compañero (82.3\%) y los problemas de acceso al empleo a los que nos hemos referido, que hacen mucho más difícil a las mujeres el poder afrontar y salir de estas situaciones.

Estas consideraciones se completan con algunos juicios formulados por los analizadores sociales en las respuestas abiertas de las entrevistas y en los grupos de discusión. La totalidad de las personas entrevistadas coinciden en señalar, como uno de los principales problemas de las mujeres de las cuencas, su baja autoestima y la infravaloración de su función social y familiar.

Las propuestas que se proponen para abordar estos problemas son las siguientes:

- En relación con los problemas laborales, se estima que son necesarias más expectativas de trabajo, más programas de formación y cualificación para la mujer, mayores incentivos a las empresas para la contratación de mujeres y para su introducción en sectores que están muy "masculinizados". En este sentido, se proponen más campañas de concienciación sobre la igualdad de la mujer; incrementar las primas a las empresas que contraten a personas pertenecientes a sectores desfavorecidos; impulsar cursos de formación y programas de orientación para el trabajo, con el fin de facilitar el acceso de la mujer al empleo; incrementar la dotación presupuestaria que se concede a los programas de la mujer; crear un mayor número de guarderías públicas y gratuitas; impulsar programas de inserción sociolaboral de las mujeres, así como programas específicos de atención a mujeres con cargas familiares y para familias monoparentales; mejorar los recursos socioeducativos y laborales de apoyo a las mujeres (educadores de apoyo, programas de desarrollo de la autoestima; superación de estereotipos, etc.).

- En relación con los problemas familiares, de convivencia o de malos tratos, se insiste en la necesidad de concienciar a la sociedad en general y a las mujeres en particular, para que éstas acudan por sí mismas a pedir ayuda a las instituciones y a denunciar las situaciones de violencia familiar. Por lo tanto, se considera adecuado incidir en una educación no sexista, que debería iniciarse en los primeros cursos escolares; potenciar más la información en temas de violencia, mediante programas, campañas informativas y divulgativas que promuevan el desarrollo de la autoestima en las mujeres; propiciar estancias más largas en los centros de acogida; conceder más ayudas y subvenciones para la adquisición o alquiler de viviendas; etc. 


\section{Problemas de las personas mayores}

Los problemas de los mayores ocupan el cuarto lugar entre las prioridades establecidas por todos los participantes en el estudio. Los analizadores sociales que respondieron a este apartado especifican los problemas que consideran más importantes de este colectivo, pero su grado de acuerdo interno es menor que en otras áreas. Desde su punto de vista, el principal problema de las personas mayores es la necesidad de orientación para favorecer un uso positivo de su tiempo libre (84.6\%). También, la mayoría destaca sus problemas de vulnerabilidad (69.3\%) y soledad (77\%). En las entrevistas se alude con detalle a la situación de cambio que se está experimentando en el cuidado de los mayores. Tradicionalmente han sido los hijos los que se han encargado de cuidar a los padres cuando éstos se hacían mayores, pero ahora, bien porque los hijos trabajan fuera o porque no quieren o pueden atenderlos, se está produciendo un aumento del número de personas mayores que viven solas, lo que a su vez hace incrementar la demanda de servicios sociales de ayuda a domicilio (Fig. 4).

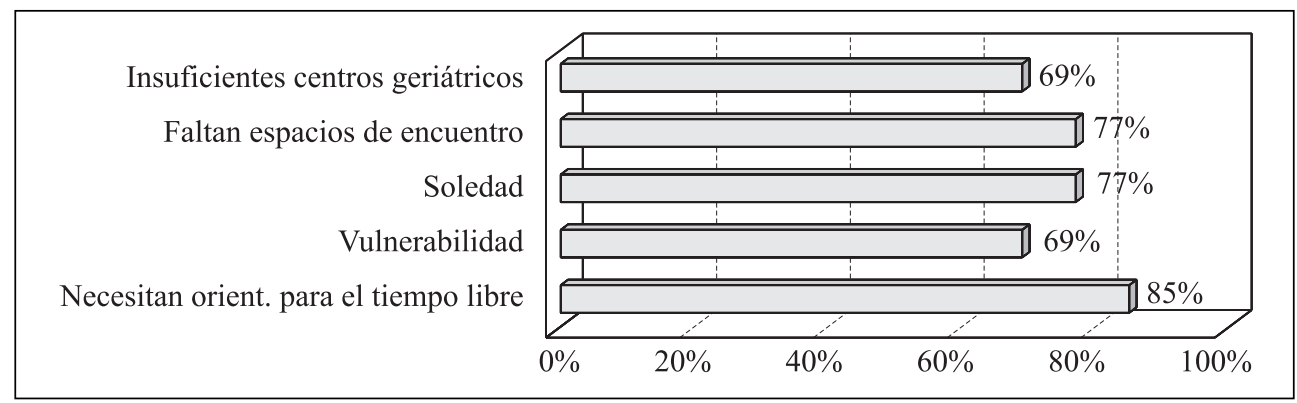

FIGURA 4.

Problemas de las personas mayores.

Asimismo, se destacan otros problemas que alcanzan porcentajes muy significativos: la necesidad de espacios de encuentro para mayores (77\%) y la insuficiencia de centros de atención geriátrica en las cuencas mineras (69.3\%). Este aspecto es preocupante, si se tiene en cuenta el progresivo envejecimiento de la población de estas zonas y las dificultades de acceso a servicios de atención y de ocio que tienen los habitantes de los lugares más periféricos, especialmente en el suroccidente de Asturias. Ello produce, en muchos casos, situaciones de masificación y largas listas de espera.

Las propuestas que se realizan para hacer frente a los problemas de los mayores son:

- Incrementar los servicios (residencias, centros de día, programas específicos) y las plantillas de profesionales que trabajan con personas mayores.

- Incrementar la participación del voluntariado en este campo, muy necesaria para dinamizar los centros y desarrollar actividades de diverso tipo.

- Introducir medidas que favorezcan una mayor coordinación entre los servicios sociales, sanitarios, voluntariado etc., con el fin de evitar que haya personas desatendidas.

- Favorecer la aportación de los mayores en programas intergeneracionales y en tareas de voluntariado. Además de la función social que ello implica (se favorecería el desa- 
rrollo personal y la integración social de este colectivo), se contribuiría a eliminar estereotipos y a que sectores de la sociedad dejaran de verlos como consumidores de recursos y de prestaciones sociales.

- Potenciar las actividades de ocio y de tiempo libre entre los mayores, no sólo desde los servicios sociales, también desde los centros educativos, escuelas de padres, etc.

\section{Problemas de las personas con discapacidad}

Los problemas de las personas con discapacidad ocupan el quinto lugar entre las prioridades que establecen los analizadores sociales en el conjunto de problemas estudiados. Sin embargo, el grado de acuerdo sobre la importancia concedida a los diferentes ítems es mayor que en otros ámbitos y a la mayoría de problemas formulados se les concede bastante relevancia.

Como en otros casos, los problemas que más preocupan tienen que ver con las enormes dificultades de acceso al mercado laboral de estos colectivos. Todos los analizadores coinciden en señalar los importantes problemas de formación que existen y su escaso nivel de cualificación para el empleo (100\%). Como consecuencia de ello, se plantea la necesidad de establecer medidas de orientación para el trabajo (90\%); de desarrollar programas de formación y servicios de asesoramiento y apoyo (80\%) y de luchar contra el efecto negativo de los extendidos estereotipos sociales sobre las personas con discapacidad, sobre sus posibilidades y sobre su contribución al mundo laboral (90\%).

El segundo grupo de problemas destacados, en estrecha conexión con los anteriores, refleja situaciones muy negativas de las personas con discapacidad en su relación con el mercado de trabajo: se considera que sus niveles de desempleo son muy altos $(80 \%)$; que les falta experiencia laboral, lo que dificulta enormemente su acceso al empleo (80\%) y que sus niveles de inactividad también son muy elevados: no sólo no tienen empleo, sino que muchos han desistido ya de intentar incorporarse al mundo laboral $(70 \%)$.

También la mayoría de los entrevistados señalan otro tipo de problemas, a los que se les atribuye, sin embargo, un nivel de importancia algo inferior a los expuestos anteriormente: problemas de dependencia familiar de las personas con discapacidad (60\%) y falta de información, apoyo y ayuda a los familiares de estos colectivos (60\%). Existe una coincidencia general entre las respuestas que los analizadores sociales dan a las preguntas cerradas y a las preguntas abiertas, si bien, en estas últimas, se alude más específicamente a las dificultades derivadas de las barreras arquitectónicas: escaleras, accesos reducidos, malas comunicaciones, etc.

Las principales medidas que se deberían adoptar para afrontar estos problemas son:

- Realizar una mayor inversión de recursos materiales y humanos para fomentar la integración humana, social y laboral del colectivo y adaptar la formación a las capacidades de estas personas.

- Introducir medidas efectivas para luchar contra los estereotipos de personas con discapacidad, que frecuentemente son vistas como no aptas para el mundo laboral. En este sentido, se plantea la necesidad de sensibilizar adecuadamente a los empresarios. 
- Crear nuevos Centros Especiales de Empleo y estructurar convenientemente el proceso formativo-ocupacional y el sistema que deberían seguir las personas con discapacidad: Centro Ocupacional - Centro Especial de Empleo - Empleo con Apoyo.

- Implantar Centros de Orientación específicos para las familias de personas con discapacidad.

- Suprimir barreras arquitectónicas.

\section{Implicaciones prácticas: propuestas socioeducativas}

El cuestionario que se aplicó a los analizadores sociales, incluía, además de las cuestiones específicas relacionadas con su perfil formativo, laboral o de implicación social, un apartado final de Problemas Generales, en el que debían valorar y explicitar problemas de diversa índole, de acuerdo con las dimensiones del cuestionario abordadas anteriormente, así como proponer medidas socioeducativas para afrontarlos de forma satisfactoria. En relación con los problemas de las cuencas mineras, los resultados de este apartado genérico añaden algunos matices de interés a las valoraciones anteriormente expuestas, aunque quizás lo más significativo sean las propuestas formuladas. Entre las medidas más relevantes que se apuntan, cabe destacar las siguientes:

- Creación de empleo (considerada la medida más importante), pues en la solución de este problema se sitúa la clave para abordar satisfactoriamente otras muchas cuestiones: se frenaría el éxodo masivo de la población hacia otras zonas, se daría un impulso a la natalidad, el sector servicios aumentaría, etc. En este sentido, se considera necesario propiciar la atracción de empresas públicas y privadas hacia el territorio, así como crear más suelo industrial y más barato y la consecución de más Fondos Mineros para la comarca.

- Un mayor apoyo institucional a las nuevas iniciativas empresariales: conceder subvenciones a los emprendedores, aprovechar de forma más efectiva los recursos que existen en la zona; llevar a cabo campañas de motivación para difundir el autoempleo; buscar nuevas alternativas en el sector turístico, maderero, ganadero; crear centros de orientación para jóvenes con el fin de que puedan crear sus propias empresas y cooperativas; realizar estudios de captación de fuentes de riqueza en relación con futuras bolsas de empleo; potenciar la actividad de ciertos sectores en la zona, como el turismo rural, las actividades al aire libre, patrimonio, parque eólico, etc.

- Adecuar la formación a las nuevas demandas del mercado laboral. Por ello se considera básico mejorar la Formación Profesional (y ampliar el número de especialidades existentes en las cuencas) con el fin de adaptarla a las necesidades del mercado laboral.

- Crear centros y programas de atención familiar, orientación para padres y jóvenes (educación en valores, prevención de consumo de drogas, de riesgos sexuales, de abuso del alcohol, etc.).

- Mejorar las vías de comunicación y los transportes públicos (itinerarios, horarios, etc.). Esta medida es reiteradamente planteada en la zona suroccidental del Principado de Asturias: en los concejos de Cangas de Narcea y Tineo y, de un modo especial, en Degaña e Ibias. 
- Aumentar la inversión económica en infraestructuras y actividades culturales, deportivas y de ocio.

Finalmente, quisimos completar estas medidas socioeducativas formuladas por los analizadores sociales, con otras propuestas planteadas por el equipo investigador, que sometimos a su consideración. Todas ellas fueron consideradas bastante o muy necesarias por más del $70 \%$ de los encuestados.

1. Sin perder de vista la elevada importancia que atribuyen a todas estas propuestas, las más demandadas son:

a) ofertar Ciclos Formativos de Formación Profesional adaptados a las necesidades del mercado laboral y a los nuevos yacimientos de empleo (89.5\%); y

b) organizar programas de cualificación profesional para jóvenes de bajo nivel de formación o que no han finalizado la educación obligatoria, como programas de Garantía Social, Escuelas Taller y Casas de Oficios (87.5\%).

2. Al siguiente grupo de propuestas les atribuyen un grado de necesidad muy similar: centros y programas de formación y orientación de mujeres para abordar los problemas específicos de este sector de población (escasa formación, dificultades de incorporación al mercado laboral, prevención de violencia y malos tratos...) (82.3\%); programas educativos para jóvenes y familias ante problemáticas específicas (fracaso escolar, consumo de drogas, incomunicación padres-hijos...) (80.4\%); nuevas especialidades formativas de cursos de formación y cualificación de desempleados (80.4\%); y programas educativos y fomento de actividades que favorezcan un uso más positivo del tiempo libre en los jóvenes (centros culturales, bibliotecas, talleres, actividades deportivas...) (80.4\%). Casi al mismo nivel, demandan centros de orientación familiar para asesorar a las familias en la prevención y actuación ante diversos problemas (fracaso escolar, consumo de drogas, incomunicación entre padres e hijos, etc.) (79.8\%); el desarrollo de centros y programas dirigidos a jóvenes con riesgo de marginación social (situaciones de abandono familiar, fracaso escolar, pobreza, drogadicción, delincuencia, etc.) (78.2\%); y el establecimiento de centros y programas de formación y orientación de personas con discapacidad (78.2\%).

3. Finalmente, en último lugar, pero también con un alto nivel de demanda, subrayan la necesidad de programas educativos para adultos (alfabetización, graduado escolar...) y el fomento de actividades que favorezcan un uso más positivo del tiempo libre en adultos, desempleados, prejubilados, personas mayores... (71.4\%).

Para terminar, desde estas páginas queremos agradecer a todas las personas que participaron en este estudio su tiempo y colaboración. Su compromiso con el desarrollo de esta región en crisis y el esfuerzo y trabajo que realizan en los distintos ámbitos de la realidad sociolaboral ya están contribuyendo a generar los cambios necesarios. Estos cambios sin embargo, exigen aún un compromiso mucho mayor de los responsables de las políticas educativas y sociales con la formación y la orientación en las cuencas mineras de Asturias.

\section{Referencias bibliográficas}

De Miguel, M. y cols. (2005). Análisis de necesidades sociales y educativas de las cuencas mineras del Principado de Asturias. Oviedo: KRK Ediciones. 
Diario "La nueva España" (Edición de las Cuencas del Nalón y Caudal) (1 de enero de 1999-30 de junio de 2001).

Diario "La nueva España" (Edición regional) (1 de enero de1999-30 de junio de 2001).

Diario "La voz de Asturias" (1 de enero de 1999-30 de junio de 2001).

Mc Killip, J. (1987). Need analysis. Tools for the Human Services and Education. Thousand Oaks CA: SAGE Publications.

Neuber, K. A. y cols. (1980). Needs Assessment. A model for community planning. Newbury Park CA, SAGE Publications.

Percy-Smith, J. (1996). Needs assessments in public policy. Buckingham, Open University Press.

Pereira, M., Pascual, J. (2004). "Metodología del estudio de análisis de las demandas y necesidades socio-educativas de las Cuencas Mineras de Asturias". Revista de Investigación Educativa, 22 (2), 353-376.

Porteus, D. (1996). "Methodologies for needs assessment", en Percy-Smith, J. (Eds.) Needs assessments in public policy. Buckingham: Open University Press.

Soriano, F. I. (1995). Conducting Needs Assessment. Thousand Oak, CA, SAGE Publications.

Stufflebeam, D. y cols. (1985). Conducting Educational Needs Assessment. Boston, Kluwer-Nighott.

Tejedor, F. J. (1990). "Perspectiva metodológica del diagnostico y evaluación de necesidades en el ámbito educativo". Revista de Investigación Educativa, (8), 16, 15-37.

Wiener, R. L. y cols. (1994). "Needs assessment. Combining Qualitative Interviews and Concept Mapping Methodology". Evaluation Review, 18, 2, 227-240.

Witkin, B. R. (1994). "Needs assessment since 1981: the state of the practice". Evaluation Practice, $15,1,17-27$.

Witkin,B. R. y Altschuld, J. W. (1995). Planning and conducting needs assessments. A practical guide. Thousand Oaks, CA: SAGE Publications.

Fecha de recepción: 04-07-05

Fecha de revisión: 03-07-06

Fecha de aceptación: 17-10-06 